\title{
Permutation resolutions for Specht modules
}

\author{
Robert Boltje · Robert Hartmann
}

Received: 23 July 2010 / Accepted: 7 November 2010 / Published online: 30 November 2010

(C) The Author(s) 2010. This article is published with open access at Springerlink.com

\begin{abstract}
For every composition $\lambda$ of a positive integer $r$, we construct a finite chain complex whose terms are direct sums of permutation modules $M^{\mu}$ for the symmetric group $\mathfrak{S}_{r}$ with Young subgroup stabilizers $\mathfrak{S}_{\mu}$. The construction is combinatorial and can be carried out over every commutative base ring $k$. We conjecture that for every partition $\lambda$ the chain complex has homology concentrated in one degree (at the end of the complex) and that it is isomorphic to the dual of the Specht module $S^{\lambda}$. We prove the exactness in special cases.
\end{abstract}

Keywords Symmetric group $\cdot$ Permutation module $\cdot$ Specht module $\cdot$ Resolution

\section{Introduction}

In the representation theory of the symmetric group, the Specht modules play a central role. They can be defined over any commutative base ring. Over a field of characteristic zero, they are precisely the simple modules, and over a field of positive characteristic the simple modules can be extracted from them. Quite a few questions concerning the Specht modules are still unanswered. The Specht modules arise as submodules of transitive permutation modules. More precisely, for every partition $\lambda=\left(\lambda_{1}, \ldots, \lambda_{l}\right)$, the corresponding Specht module $S^{\lambda}$ arises as a submodule of the permutation module $M^{\lambda}$ whose point stabilizer is the Young subgroup $\mathfrak{S}_{\lambda}$ which is

R. Boltje's research partially supported by NSF Grant DMS 0200592.

R. Boltje ( $\varangle)$

Department of Mathematics, University of California, Santa Cruz, CA 95064, USA

e-mail: boltje@ucsc.edu

R. Hartmann

Department of Mathematics, University of Stuttgart, Pfaffenwaldring 57, 70569 Stuttgart, Germany

e-mail: Robert.Hartmann@mathematik.unistuttgart.de 
isomorphic to $\mathfrak{S}_{\lambda_{1}} \times \cdots \times \mathfrak{S}_{\lambda_{l}}$. Over fields of characteristic 0 , the multiplicity of $S^{\lambda}$ as a direct summand in $M^{\mu}$ is equal to the number of generalized standard tableaux of shape $\lambda$ and content $\mu$ (Young's rule). This is only one instance of the combinatorial flavor that pervades the representation theory of the symmetric group. The goal of this paper is, for every partition $\lambda$, the combinatorial construction of a chain complex that consists of direct sums of permutation modules $M^{\mu}$ and that is a resolution of the Specht module $S^{\lambda}$. The nature of our construction will actually force us to use the dual of the Specht module to augment the constructed chain complex. More precisely, we construct a finite chain complex $C_{*}^{\lambda}$ which can be extended by one term, namely the dual of $S^{\lambda}$. We denote this augmented chain complex by $\tilde{C}_{*}^{\lambda}$. One can always switch to the dual of the chain complex to have $S^{\lambda}$ as the initial term, if desired. The construction of the chain complex works in greater generality, namely for every composition $\lambda$. However, we can see in examples that it does not always have homology concentrated in one degree. For partitions, we conjecture that the chain complex is exact over arbitrary base rings. We prove in general that the complex is exact in the two final degrees and we prove that the complex is exact for every tame composition. Tameness is a technical condition which makes the inductive proof work. Tame compositions include partitions with at most two parts and partitions of the form $\left(\lambda_{1}, \lambda_{2}, 1\right)$.

Let us compare our resolution with similar complexes appearing in the literature. The first related results were independently obtained by Akin [1] and Zelevinskii [14]. Their approach was quite different. They construct resolutions of Weyl modules for the general linear group. Under the Schur functor, such a resolution yields a resolution of the corresponding Specht module for the symmetric group, cf. [14, Example 3]. However, the Akin-Zelevinskii resolution is constructed over the complex numbers and will, in general, not be exact over other commutative rings. There were some attempts to generalize this result to positive characteristic, and we will compare these with our resolution in the final section.

The paper is arranged as follows. In Sect. 2, we introduce the notation and terminology that is used throughout the paper, and we motivate the construction of the chain complex by deriving an alternating sum formula for the Specht module in terms of permutation modules. The chain complex is constructed in Sect. 3, and exactness in degrees -1 and 0 is proven in Sect. 4. Section 5 introduces and studies quasipartitions and tame compositions. In Sect. 6 , we prove that the chain complex $\tilde{C}_{*}^{\lambda}$ is exact if $\lambda$ is a tame composition. The last section gives an overview on related results.

The authors would like to express their gratefulness to the referee and to Bhama Srinivasan for making them aware of several related papers that are discussed in Sect. 7.

\section{Notation and motivation}

Throughout this paper, we fix a positive integer $r$ and a commutative base ring $k$. The symmetric group on the set $\{1, \ldots, r\}$ is denoted by $\mathfrak{S}_{r}$ and its group algebra over $k$ is denoted by $k \mathfrak{S}_{r}$. Our convention for the multiplication in $\mathfrak{S}_{r}$ is that $\mathfrak{S}_{r}$ consists of functions, and that the composition $\sigma \tau$ of two elements $\sigma, \tau \in \mathfrak{S}_{r}$ is defined by first 
applying $\tau$ and then $\sigma$. Unadorned tensor products or homomorphisms are meant to be formed over $k$.

\section{1}

Recall that a composition of $r$ is a sequence $\lambda=\left(\lambda_{1}, \lambda_{2}, \ldots\right)$ of non-negative integers $\lambda_{i}$ with the property that only finitely many of them are non-zero and that their sum is equal to $r$. If $\lambda_{l}$ is the last non-zero element in the sequence $\lambda$ then $l(\lambda):=l$ is called the length of $\lambda$. We also write $\lambda=\left(\lambda_{1}, \ldots, \lambda_{n}\right)$ for any $n \geq l(\lambda)$. If $\lambda_{1} \geq$ $\lambda_{2} \geq \lambda_{3} \geq \cdots$ then $\lambda$ is called a partition of $r$. We denote the set of compositions (resp., partitions) of $r$ by $\Gamma_{r}$ (resp., $\Lambda_{r}$ ). Thus, $\Lambda_{r}$ is a finite subset of the infinite set $\Gamma_{r}$. The dominance partial order on $\Gamma_{r}$ is defined by

$$
\lambda \unlhd \mu: \quad \Longleftrightarrow \sum_{i=1}^{e} \lambda_{i} \leq \sum_{i=1}^{e} \mu_{i} \quad \text { for all } e \geq 1 .
$$

Note that although $\Gamma_{r}$ is infinite, for any given $\lambda \in \Gamma_{r}$, the set $\Gamma_{r}^{\unrhd \lambda}$ of all elements $\mu \in \Gamma_{r}$ with $\mu \unrhd \lambda$ is finite.

For $\lambda \in \Gamma_{r}$, the corresponding Young subgroup $\mathfrak{S}_{\lambda}$ of $\mathfrak{S}_{r}$ is defined as the set of all permutations in $\mathfrak{S}_{r}$ which stabilize the intervals $\left\{1, \ldots, \lambda_{1}\right\},\left\{\lambda_{1}+1, \ldots, \lambda_{1}+\lambda_{2}\right\}$, $\ldots$ of $\{1, \ldots, r\}$. The Young diagram of $\lambda=\left(\lambda_{1}, \lambda_{2}, \ldots\right)$ is the left aligned array of a row of $\lambda_{1}$ square boxes, followed by a row of $\lambda_{2}$ square boxes, etc. For instance, the Young diagram of $\lambda=(1,0,2)$ is

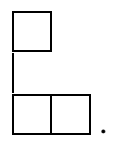

We often identify compositions with their Young diagrams and call $\lambda$ the shape of its corresponding Young diagram. We also number the $r$ boxes of the Young diagram of shape $\lambda$ by going from left to right in the first row, then from left to right in the second row, and so on.

\section{2}

Let $\lambda, \mu \in \Gamma_{r}$. A generalized tableau of shape $\lambda$ and content $\mu$ is a filling of the $r$ boxes of the Young diagram of shape $\lambda$ with positive integers such that for every positive integer $i$ there are precisely $\mu_{i}$ entries that are equal to $i$. More formally, for fixed shape $\lambda$, one can define a generalized tableau of shape $\lambda$ and content $\mu$ as a function $T:\{1, \ldots, r\} \rightarrow \mathbb{N}$ such that $\left|T^{-1}(i)\right|=\mu_{i}$ for all $i \in \mathbb{N}$. We visualize $T$ as the Young diagram of shape $\lambda$ with entry $T(p)$ in its $p$ th box. The set of generalized tableaux of shape $\lambda$ and content $\mu$ is denoted by $\mathcal{T}(\lambda, \mu)$. The set $\mathcal{T}(\lambda, \mu)$ has a total (lexicographic) order given by $T<T^{\prime}$ if and only if, for the smallest $p \in\{1, \ldots, r\}$ with $T(p) \neq T^{\prime}(p)$, we have $T(p)<T^{\prime}(p)$. The smallest element in $\mathcal{T}(\lambda, \mu)$ is denoted by $T_{\mu}^{\lambda}$. For instance,

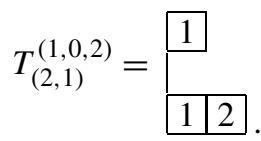


A generalized tableau $T \in \mathcal{T}(\lambda, \mu)$ is called row-semistandard if in each row of $T$ the entries are weakly increasing from left to right. It is called standard if additionally $\lambda$ is a partition and the entries in the columns of $T$ are strictly increasing from top to bottom. The set of row-semistandard (resp., standard) $\lambda$-tableaux of content $\mu$ is denoted by $\mathcal{T}^{\mathrm{rs}}(\lambda, \mu)$ (resp., $\left.\mathcal{T}^{\mathrm{st}}(\lambda, \mu)\right)$. Thus, $\mathcal{T}^{\mathrm{st}}(\lambda, \mu) \subseteq \mathcal{T}^{\mathrm{rs}}(\lambda, \mu) \subseteq \mathcal{T}(\lambda, \mu)$ and $\mathcal{T}^{\text {st }}(\lambda, \mu)=\emptyset$ if $\lambda \notin \Lambda_{r}$.

The group $\mathfrak{S}_{r}$ acts transitively from the right on $\mathcal{T}(\lambda, \mu)$ by place permutations: For $T \in \mathcal{T}(\lambda, \mu)$ and $\sigma \in \mathfrak{S}_{r}$, we define $T \sigma$ by $(T \sigma)(p)=T(\sigma(p))$, where $p \in$ $\{1, \ldots, r\}$. In other words, $T \sigma$ arises from $T$ by shifting the entry in box $q$ of $T$ to box $\sigma^{-1}(q)$, for all $q \in\{1, \ldots, r\}$. The stabilizer of $T_{\mu}^{\lambda}$ is equal to $\mathfrak{S}_{\mu}$, and we have an isomorphism

$$
\mathfrak{S}_{\mu} \backslash \mathfrak{S}_{r} \stackrel{\sim}{\longrightarrow} \mathcal{T}(\lambda, \mu), \quad \mathfrak{S}_{\mu} \sigma \mapsto T_{\mu}^{\lambda} \sigma
$$

of right $\mathfrak{S}_{r}$-sets.

We call two elements $T, T^{\prime} \in \mathcal{T}(\lambda, \mu)$ row-equivalent if $T^{\prime}$ arises from $T$ by rearranging the entries of $T$ within each row (in other words, if $T^{\prime}=T \sigma$ for some $\left.\sigma \in \mathfrak{S}_{\lambda}\right)$. We write $\{T\}$ for the row-equivalence class of $T$ and we denote the set of row-equivalence classes of $\mathcal{T}(\lambda, \mu)$ by $\overline{\mathcal{T}}(\lambda, \mu)$. The bijection in (2.1) induces the bijection

$$
\mathfrak{S}_{\mu} \backslash \mathfrak{S}_{r} / \mathfrak{S}_{\lambda} \stackrel{\sim}{\longrightarrow} \overline{\mathcal{T}}(\lambda, \mu), \quad \mathfrak{S}_{\mu} \sigma \mathfrak{S}_{\lambda} \mapsto\left\{T_{\mu}^{\lambda} \sigma\right\}
$$

Since every row-equivalence class of $\mathcal{T}(\lambda, \mu)$ contains a unique row-semistandard tableau, we also have a bijection

$$
\mathcal{T}^{\mathrm{rs}}(\lambda, \mu) \stackrel{\sim}{\longrightarrow} \overline{\mathcal{T}}(\lambda, \mu), \quad T \mapsto\{T\}
$$

so that we obtain a resulting bijection

$$
\mathcal{T}^{\mathrm{rs}}(\lambda, \mu) \cong \mathfrak{S}_{\mu} \backslash \mathfrak{S}_{r} / \mathfrak{S}_{\lambda}
$$

In the case that $\mu=(1, \ldots, 1)$, we will denote generalized $\lambda$-tableaux with content $\mu$ by lower case letters, e.g., $t$ instead of $T$, and just call them $\lambda$-tableaux without reference to their content. We also write $t^{\lambda}, \mathcal{T}(\lambda), \mathcal{T}^{\mathrm{rs}}(\lambda), \mathcal{T}^{\text {st }}(\lambda), \overline{\mathcal{T}}(\lambda)$ instead of $T_{\mu}^{\lambda}, \mathcal{T}(\lambda, \mu), \mathcal{T}^{\mathrm{rs}}(\lambda, \mu), \mathcal{T}^{\mathrm{st}}(\lambda, \mu), \overline{\mathcal{T}}(\lambda, \mu)$, respectively.

Besides the right $\mathfrak{S}_{r}$-action introduced above, the set $\mathcal{T}(\lambda)$ has also a transitive free left action of $\mathfrak{S}_{r}$ defined by $(\sigma t)(p):=\sigma(t(p))$ for $t \in \mathcal{T}(\lambda), \sigma \in \mathfrak{S}_{r}$ and $p \in$ $\{1, \ldots, r\}$. These two actions commute, and the isomorphism of right $\mathfrak{S}_{r}$-sets in (2.1) becomes an isomorphism

$$
\mathfrak{S}_{r} \stackrel{\sim}{\longrightarrow} \mathcal{T}(\lambda), \quad \sigma \mapsto t^{\lambda} \sigma,
$$

of left and right $\mathfrak{S}_{r}$-sets. It induces an isomorphism

$$
\mathfrak{S}_{r} / \mathfrak{S}_{\lambda} \stackrel{\sim}{\longrightarrow} \overline{\mathcal{T}}(\lambda), \quad \sigma \mathfrak{S}_{\lambda} \mapsto\left\{t^{\lambda} \sigma\right\},
$$

of left $\mathfrak{S}_{r}$-sets, cf. (2.2). Finally, using the special case of the bijection (2.3), we obtain bijections

$$
\mathcal{T}^{\mathrm{rs}}(\lambda) \cong \overline{\mathcal{T}}(\lambda) \cong \mathfrak{S}_{r} / \mathfrak{S}_{\lambda}
$$


We will often identify these three sets via these canonical bijections and we will consider $\mathcal{T}^{\mathrm{rs}}(\lambda)$ as a left $\mathfrak{S}_{r}$-set through this identification.

\section{3}

Let $\lambda \in \Gamma_{r}$. We denote by $M^{\lambda}$ the permutation $k \mathfrak{S}_{r}$-module with $k$-basis $\overline{\mathcal{T}}(\lambda)$ and the left $\mathfrak{S}_{r}$-action defined in Sect. 2.2. We use the identification (2.5) and view $M^{\lambda}$ also as the free $k$-module with basis $\mathcal{T}^{\mathrm{rs}}(\lambda)$. Note that the map

$$
\operatorname{Ind}_{\mathfrak{S}_{\lambda}}^{\mathfrak{S}_{r}}(k)=k \mathfrak{S}_{r} \otimes_{k \mathfrak{S}_{\lambda}} k \rightarrow M^{\lambda}, \quad \sigma \otimes 1 \mapsto \sigma t^{\lambda}
$$

is an isomorphism of left $k \mathfrak{S}_{r}$-modules, where $k$ stands for the trivial $k \mathfrak{S}_{\lambda}$-module. The Specht module $S^{\lambda}$ is defined as the $k$-span of the elements

$$
e_{t}:=\kappa_{t} \cdot t \in M^{\lambda}, \quad t \in \mathcal{T}^{\mathrm{st}}(\lambda),
$$

where $\kappa_{t}:=\sum_{\sigma \in C_{t}} \operatorname{sgn}(\sigma) \sigma \in k \mathfrak{S}_{r}$ and $C_{t} \leq \mathfrak{S}_{r}$ denotes the column stabilizer of the $\lambda$-tableau $t$. It follows from standard arguments (see, for instance, [10, $\S 4$ and $\S 8]$ ) that the elements $e_{t} \in M^{\lambda}, t \in \mathcal{T}^{\text {st }}(\lambda)$, are $k$-linearly independent and that their $k$-span is a $k \mathfrak{S}_{r}$-submodule of $M^{\lambda}$. Note that by definition we have $S^{\lambda}=0$ if $\lambda$ is not a partition. It is easy to see (cf. [10, Lemma 8.3]) that for every $t \in \mathcal{T}^{\text {st }}(\lambda)$, the element $e_{t} \in S^{\lambda}$ can be written as

$$
e_{t}=t+\sum_{t<s \in \mathcal{T}^{\mathrm{rs}}(\lambda)} \alpha_{s} \cdot s
$$

with elements $\alpha_{s} \in k$.

For this subsection, we assume that $k$ is a field of characteristic 0 . Then the Specht modules $S^{\lambda}, \lambda \in \Lambda_{r}$, form a set of representatives for the isomorphism classes of irreducible $k \mathfrak{S}_{r}$-modules. In other words, their associated elements $\left[S^{\lambda}\right]$ in the Grothendieck group $R\left(k \mathfrak{S}_{r}\right)$ of finitely generated left $k \mathfrak{S}_{r}$-module form the canonical $\mathbb{Z}$-basis of the abelian group $R\left(k \mathfrak{S}_{r}\right)$, cf. [10, 111$]$. Young's rule (cf. $\left.[10, \S 14]\right)$ states that, for every $\lambda \in \Lambda_{r}$, one has the equation

$$
\left[M^{\lambda}\right]=\sum_{\lambda \unlhd \mu \in \Lambda_{r}} \kappa_{\lambda, \mu}\left[S^{\mu}\right]
$$

in $R\left(k \mathfrak{S}_{r}\right)$, with $\kappa_{\lambda, \mu}$, the Kostka numbers, defined by

$$
\kappa_{\lambda, \mu}:=\left|\mathcal{T}^{\mathrm{st}}(\mu, \lambda)\right|
$$

Since $\kappa_{\lambda, \lambda}=1$, for all $\lambda \in \Lambda_{r}$, the elements $\left[M^{\lambda}\right], \lambda \in \Lambda_{r}$, form also a $\mathbb{Z}$-basis of $R\left(k \mathfrak{S}_{r}\right)$. The transformation matrix $X=\left(\kappa_{\lambda, \mu}\right)$ between these two bases is upper triangular with diagonal entries equal to 1 (if one uses a suitable ordering of the elements in $\Lambda_{r}$ ). Thus, $X=I+Y$, where $I$ denotes the identity matrix and $Y$ is a 
nilpotent matrix with $(\lambda, \mu)$-entry equal to $\kappa_{\lambda, \mu}$ if $\lambda \triangleleft \mu$ and equal to 0 otherwise. An easy induction argument on $n$ shows that the $(\lambda, \mu)$-entry of $Y^{n}$ is equal to

$$
\sum_{\lambda=\lambda^{(0)} \triangleleft \lambda^{(1)} \triangleleft \cdots \triangleleft \lambda^{(n)}=\mu} \kappa_{\lambda^{(0)}, \lambda^{(1)}} \kappa_{\lambda^{(1)}, \lambda^{(2)}} \cdots \kappa_{\lambda^{(n-1)}, \lambda^{(n)}},
$$

where the sum runs over all strictly increasing chains of length $n$ in the partially ordered set $\Lambda_{r}$ that start with $\lambda$ and end with $\mu$. Since $Y$ is nilpotent, we have $(I+$ $Y)\left(I-Y+Y^{2}-Y^{3}+\cdots\right)=I$. This implies $X^{-1}=I-Y+Y^{2}-Y^{3}+\cdots$, and we obtain

$$
\left[S^{\lambda}\right]=\sum_{\lambda=\lambda^{(0)} \triangleleft \lambda^{(1)} \triangleleft \cdots \triangleleft \lambda^{(n)}}(-1)^{n} \kappa_{\lambda^{(0)}, \lambda^{(1)}} \kappa_{\lambda^{(1)}, \lambda^{(2)}} \cdots \kappa_{\lambda^{(n-1)}, \lambda^{(n)}}\left[M^{\lambda^{(n)}}\right]
$$

in $R\left(K \mathfrak{S}_{r}\right)$, for every $\lambda \in \Lambda_{r}$. Here, the sum runs over all strictly ascending chains in $\Lambda_{r}$ that start with $\lambda$.

The motivation for this paper comes from (2.10). Our goal is to construct a chain complex, using the modules $M^{\mu}$, which mimics the right hand side of this equation in the sense that its degree $n$ term contributes precisely the part of the sum which comes from chains of length $n$. Ideally, we want that this chain complex is a resolution of $S^{\lambda}$. To this end, it is useful to interpret the occurring multiplicities

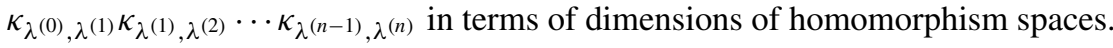

\section{5}

Let $k$ be again an arbitrary commutative ring. Using the explicit isomorphism (2.6), the Mackey decomposition formula (cf. [5, Theorem 10.13]), and the adjointness of induction and restriction, we obtain explicit and canonical isomorphisms of $k$ modules,

$$
\begin{aligned}
\operatorname{Hom}_{k \mathfrak{S}_{r}}\left(M^{\mu}, M^{\lambda}\right) & \cong \operatorname{Hom}_{k \mathfrak{S}_{r}}\left(\operatorname{Ind}_{\mathfrak{S}_{\mu}}^{\mathfrak{S}_{r}}(k), \operatorname{Ind}_{\mathfrak{S}_{\lambda}}^{\mathfrak{S}_{r}}(k)\right) \\
& \cong \operatorname{Hom}_{k \mathfrak{S}_{\mu}}\left(k, \operatorname{Res}_{\mathfrak{S}_{\mu}}^{\mathfrak{S}_{r}} \operatorname{Ind}_{\mathfrak{S}_{\lambda}}^{\mathfrak{S}_{r}}(k)\right) \\
& \cong \bigoplus_{\sigma \in \mathfrak{S}_{\mu} \backslash \mathfrak{S}_{r} / \mathfrak{S}_{\lambda}} \operatorname{Hom}_{k} \mathfrak{S}_{\mu}\left(k, \operatorname{Ind}_{\mathfrak{S}_{\mu} \cap \sigma \mathfrak{S}_{\lambda} \sigma^{-1}}^{\mathfrak{S}_{\mu}}(k)\right) .
\end{aligned}
$$

Furthermore, for $\sigma \in \mathfrak{S}_{r}$, one has an isomorphism of $k$-modules

$$
\operatorname{Hom}_{k \mathfrak{S}_{\mu}}\left(k, \operatorname{Ind}_{\mathfrak{S}_{\mu} \cap \sigma \mathfrak{S}_{\lambda} \sigma^{-1}}^{\mathfrak{S}_{\mu}}(k)\right) \cong k,
$$

under which $1 \in k$ corresponds to the homomorphism taking 1 to $\sum_{\tau \in \mathfrak{S}_{\mu} / \mathfrak{S}_{\mu} \cap \sigma \mathfrak{S}_{\lambda} \sigma^{-1}} \tau \otimes 1$. Thus, using the explicit bijection (2.4) we obtain an explicit $k$-module isomorphism

$$
\operatorname{Hom}_{k \mathfrak{S}_{r}}\left(M^{\mu}, M^{\lambda}\right) \cong \bigoplus_{\sigma \in \mathfrak{S}_{\mu} \backslash \mathfrak{S}_{r} / \mathfrak{S}_{\lambda}} k \cong k\left[\mathfrak{S}_{\mu} \backslash \mathfrak{S}_{r} / \mathfrak{S}_{\lambda}\right] \cong k \mathcal{T}^{\mathrm{rs}}(\lambda, \mu)
$$


where $k\left[\mathfrak{S}_{\mu} \backslash \mathfrak{S}_{r} / \mathfrak{S}_{\lambda}\right]$ (resp., $k \mathcal{T}^{\mathrm{rs}}(\lambda, \mu)$ ) denotes the free $k$-module with basis $\mathfrak{S}_{\mu} \backslash \mathfrak{S}_{r} / \mathfrak{S}_{\lambda}$ (resp., $\left.\mathcal{T}^{\mathrm{rs}}(\lambda, \mu)\right)$. We denote the homomorphism in $\operatorname{Hom}_{k} \mathfrak{S}_{r}\left(M^{\mu}, M^{\lambda}\right)$ which corresponds to $T \in \mathcal{T}^{\mathrm{rs}}(\lambda, \mu)$ by $\theta_{T}$. By construction, the homomorphisms $\theta_{T}, T \in \mathcal{T}^{\mathrm{rs}}(\lambda, \mu)$, form a $k$-basis of $\operatorname{Hom}_{k \mathfrak{S}_{r}}\left(M^{\mu}, M^{\lambda}\right)$. We leave it to the reader to verify the following explicit description of $\theta_{T}$ :

For $t \in \mathcal{T}^{\mathrm{rs}}(\mu)$ and $T \in \mathcal{T}^{\mathrm{rs}}(\lambda, \mu)$, the element $\theta_{T}(t) \in M^{\lambda}$ is equal to the sum of all $\lambda$-tableaux $s \in \mathcal{T}^{\mathrm{rs}}(\lambda)$ with the following property for each $i=$ $1, \ldots, l(\lambda)$ : If the ith row of $T$ contains precisely $x$ entries equal to $y$ then the ith row of $s$ contains precisely $x$ entries from the yth row of $t$.

So, for instance, one has

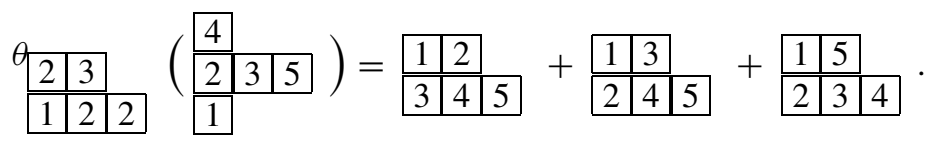

Remark 2.6 Equation (2.10) together with (2.9) and (2.11) suggests constructing, for $\lambda \in \Lambda_{r}$, a chain complex whose term in degree $n$ is given by

$$
\begin{gathered}
\bigoplus_{\lambda=\lambda^{(0)} \triangleleft \lambda^{(1)} \triangleleft \cdots \triangleleft \lambda(n)} \operatorname{Hom}_{k \mathfrak{S}_{r}}^{\text {st }}\left(M^{\lambda^{(0)}}, M^{\lambda^{(1)}}\right) \otimes \operatorname{Hom}_{k \mathfrak{S}_{r}}^{\mathrm{st}}\left(M^{\lambda^{(1)}}, M^{\lambda^{(2)}}\right) \otimes \cdots \\
\otimes \operatorname{Hom}_{k \mathfrak{S}_{r}}^{\mathrm{st}}\left(M^{\lambda^{(n-1)}}, M^{\lambda^{(n)}}\right) \otimes \operatorname{Hom}\left(M^{\lambda^{(n)}}, k\right),
\end{gathered}
$$

where $\operatorname{Hom}_{k \mathfrak{S}_{r}}^{\text {st }}\left(M^{\mu}, M^{\lambda}\right)$ denotes the $k$-span of the elements $\theta_{T}, T \in \mathcal{T}^{\text {st }}(\lambda, \mu)$, and using the composition of homomorphisms to define the boundary maps by a standard simplicial construction. Unfortunately, if $\nu \triangleleft \mu \triangleleft \lambda$ are in $\Lambda_{r}$ and $T \in \mathcal{T}^{\mathrm{st}}(\lambda, \mu)$, $U \in \mathcal{T}^{\text {st }}(\mu, v)$ then $\theta_{T} \circ \theta_{U}$ is not necessarily contained in $\operatorname{Hom}_{k \mathfrak{S}_{r}}^{\mathrm{st}}\left(M^{v}, M^{\lambda}\right)$. For instance, if we choose

$$
(3,3,3) \triangleleft(4,3,2) \triangleleft(5,4), \quad T=\begin{array}{|l|l|l|l|l|}
\hline 1 & 1 & 1 & 1 & 3 \\
\hline \hline 2 & 2 & 2 & 3
\end{array}, \quad U=\begin{array}{|l|l|l|l|}
\hline 1 & 1 & 1 & 3 \\
\hline \hline 2 & 2 & 2 \\
\hline \hline 3 & 3 &
\end{array}
$$

then $\theta_{T} \circ \theta_{U}=2 \theta_{V}$ with

$$
V=\begin{array}{|l|l|l|l|l|}
\hline 1 & 1 & 1 & 3 & 3 \\
\hline \hline 2 & 2 & 2 & 3 \\
\hline
\end{array}
$$

which is not a standard generalized tableau. We will still use the idea of a simplicial construction, but we will allow more than just the standard generalized tableaux to define Hom-sets that are closed under composition, and we will also allow chains of compositions instead of chains of partitions.

\section{Definition of the chain complex $\tilde{C}_{*}^{\lambda}$}

Throughout this section, we fix a composition $\lambda \in \Gamma_{r}$. 
3.1

Let $\mu$ and $\nu$ be compositions of $r$. We define $\mathcal{T}^{\wedge}(\mu, v) \subseteq \mathcal{T}^{\mathrm{rs}}(\mu, v)$ as the set of those generalized row-semistandard tableaux $T$ of shape $\mu$ and content $\nu$ with the property that the $i$ th row of $T$ contains no entry that is smaller than $i$, for $i=1, \ldots, l(\mu)$. Obviously, we have

$$
\mathcal{T}^{\mathrm{st}}(\mu, v) \subseteq \mathcal{T}^{\wedge}(\mu, v) \subseteq \mathcal{T}^{\mathrm{rs}}(\mu, v)
$$

and

$$
\mathcal{T}^{\wedge}(\mu, v) \neq \emptyset \Longleftrightarrow T_{v}^{\mu} \in \mathcal{T}^{\wedge}(\mu, v) \Longleftrightarrow v \unlhd \mu .
$$

We set

$$
\operatorname{Hom}_{k \mathfrak{S}_{r}}^{\wedge}\left(M^{v}, M^{\mu}\right):=\bigoplus_{T \in \mathcal{T}^{\wedge}(\mu, v)} k \theta_{T} \subseteq \operatorname{Hom}_{k \mathfrak{S}_{r}}\left(M^{v}, M^{\mu}\right) .
$$

By the explicit rule (2.12) describing how $\theta_{T}$ applies to a basis element $t \in \mathcal{T}^{\mathrm{rs}}(v)$ of $M^{v}$, we see that $T \in \mathcal{T}^{\wedge}(\mu, v)$ if and only if the constituents $s \in \mathcal{T}^{\mathrm{rs}}(\mu)$ of $\theta_{T}(t)$ arise from $t$ by moving boxes of $t$ together with their entries upwards. From this interpretation it is obvious that, for $\pi, \mu, \nu \in \Gamma_{r}$ and $T \in \mathcal{T}^{\wedge}(\mu, \nu), S \in \mathcal{T}^{\wedge}(\pi, \mu)$, one has

$$
\theta_{S} \circ \theta_{T} \in \operatorname{Hom}_{k \mathfrak{S}_{r}}^{\wedge}\left(M^{v}, M^{\pi}\right)
$$

We set

$$
\kappa_{v, \mu}^{\wedge}:=\left|\mathcal{T}^{\wedge}(\mu, v)\right|
$$

and note that

$$
\kappa_{\mu, \mu}^{\wedge}=1 \quad \text { and that } \quad \kappa_{\nu, \mu}^{\wedge}=0 \quad \text { unless } \nu \unlhd \mu .
$$

For any strictly increasing chain

$$
\gamma=\left(\lambda^{(0)} \triangleleft \cdots \triangleleft \lambda^{(n)}\right)
$$

of length $n$ in $\Gamma_{r}$, we set

$$
\begin{aligned}
M_{\gamma}:= & \operatorname{Hom}_{k \mathfrak{S}_{r}}^{\wedge}\left(M^{\lambda^{(0)}}, M^{\lambda^{(1)}}\right) \otimes \operatorname{Hom}_{k \mathfrak{S}_{r}}^{\wedge}\left(M^{\lambda^{(1)}}, M^{\lambda^{(2)}}\right) \otimes \cdots \\
& \otimes \operatorname{Hom}_{k \mathfrak{S}_{r}}^{\wedge}\left(M^{\lambda^{(n-1)}}, M^{\lambda^{(n)}}\right) \otimes \operatorname{Hom}\left(M^{\lambda^{(n)}}, k\right)
\end{aligned}
$$

and view $M_{\gamma}$ as a $k \mathfrak{S}_{r}$-module by

$$
\sigma \cdot\left(\theta_{1} \otimes \cdots \otimes \theta_{n} \otimes \epsilon\right):=\theta_{1} \otimes \cdots \otimes \theta_{n} \otimes \sigma \epsilon
$$

where

$$
(\sigma \epsilon)(m):=\epsilon\left(\sigma^{-1} m\right)
$$


for $\sigma \in \mathfrak{S}_{r}, \theta_{i} \in \operatorname{Hom}_{k \mathfrak{S}_{r}}^{\wedge}\left(M^{\lambda^{(i-1)}}, M^{\lambda^{(i)}}\right), i \in\{1, \ldots, n\}, \epsilon \in \operatorname{Hom}\left(M^{\lambda^{(n)}}, k\right)$ and $m \in$ $M^{\lambda^{(n)}}$. Thus, $M_{\gamma}$ is isomorphic to the $\kappa_{\lambda^{(0)}, \lambda^{(1)}}^{\wedge} \cdots \kappa_{\lambda^{(n-1)}, \lambda^{(n)}}^{\wedge}$-fold direct sum of copies of the $k$-dual of $M^{\lambda^{(n)}}$ (which is again isomorphic to $M^{\lambda^{(n)}}$ ).

For an integer $n \geq 0$, we denote by $\Delta_{n}^{\lambda}$ the set of chains $\gamma$ in $\Gamma_{r}$ as in (3.1) of length $n$ with $\lambda^{(0)}=\lambda$. Moreover, we set

$$
C_{n}^{\lambda}:=\bigoplus_{\gamma \in \Delta_{n}^{\lambda}} M_{\gamma}
$$

For $n \geq 1, \gamma \in \Delta_{n}^{\lambda}$ and $i \in\{1, \ldots, n\}$, we denote by $\gamma_{i} \in \Delta_{n-1}^{\lambda}$ the chain arising from $\gamma$ by omitting $\lambda^{(i)}$. We define the homomorphism

$$
d_{n, i}^{\lambda}: M_{\gamma} \rightarrow M_{\gamma_{i}}, \quad \theta_{1} \otimes \cdots \otimes \theta_{n} \otimes \epsilon \mapsto \theta_{1} \otimes \cdots \otimes \theta_{i+1} \circ \theta_{i} \otimes \cdots \otimes \epsilon,
$$

of $k \mathfrak{S}_{r}$-modules, where we interpret $\theta_{i+1}$ as $\epsilon$ if $i=n$. The direct sum of these homomorphisms defines a homomorphism $d_{n, i}^{\lambda}: C_{n}^{\lambda} \rightarrow C_{n-1}^{\lambda}$ and we set

$$
d_{n}^{\lambda}:=\sum_{i=1}^{n}(-1)^{i-1} d_{n, i}: C_{n}^{\lambda} \rightarrow C_{n-1}^{\lambda}
$$

for $n \geq 1$. By the definition of the maps $d_{n, i}^{\lambda}$, they satisfy the usual simplicial relations. Thus, $d_{n}^{\lambda} \circ d_{n+1}^{\lambda}=0$ for $n \geq 1$, and we have constructed a chain complex

$$
C_{*}^{\lambda}: \quad 0 \rightarrow C_{d(\lambda)}^{\lambda} \stackrel{d_{d(\lambda)}^{\lambda}}{\longrightarrow} C_{d(\lambda)-1}^{\lambda} \stackrel{d_{d(\lambda)-1}^{\lambda}}{\longrightarrow} \cdots \stackrel{d_{1}^{\lambda}}{\longrightarrow} C_{0}^{\lambda} \longrightarrow 0
$$

of $k \mathfrak{S}_{r}$-modules, where $d(\lambda)$ denotes the depth of $\lambda$ in $\Gamma_{r}$, i.e., the maximal number $n$ such that there exists a chain $\lambda=\lambda^{(0)} \triangleleft \cdots \triangleleft \lambda^{(n)}$ in $\Gamma_{r}$. It is not difficult to see that $d(\lambda)=\lambda_{2}+2 \lambda_{3}+3 \lambda_{4}+\cdots+(l-1) \lambda_{l}$ if $\lambda=\left(\lambda_{1}, \ldots, \lambda_{l}\right)$. But we will not use this fact. Often we will just write $d_{n}$ instead of $d_{n}^{\lambda}$.

We will denote by $\epsilon_{t} \in \operatorname{Hom}\left(M^{\lambda}, k\right), t \in \mathcal{T}^{\mathrm{rs}}(\lambda)$, the dual $k$-basis of the basis elements $t \in \mathcal{T}^{\mathrm{rs}}(\lambda)$ of $M^{\lambda}$. Thus, $\epsilon_{t}(s)=\delta_{s, t}$ for $s, t \in \mathcal{T}^{\mathrm{rs}}(\lambda)$. We leave it to the reader to verify the following explicit formula for the map $d_{1}: C_{1}^{\lambda} \rightarrow C_{0}^{\lambda}$ which follows immediately from (2.12):

For $\lambda \triangleleft \mu$ in $\Gamma_{r}, T \in \mathcal{T}^{\mathrm{rs}}(\mu, \lambda)$ and $s \in \mathcal{T}^{\mathrm{rs}}(\mu)$ one has $\epsilon_{s} \circ \theta_{T}=\sum_{t} \epsilon_{t}$, where $t$ runs through all elements in $\mathcal{T}^{\mathrm{rs}}(\lambda)$ with the following property for $i \in\{1, \ldots, l(\mu)\}$ : If the $i$ th row of $T$ contains precisely $x$ entries equal to $y$ then the $y$ th row of $t$ contains precisely $x$ entries from the ith row of $s$.

Finally, we extend the chain complex $C_{*}^{\lambda}$ by the map

$$
d_{0}^{\lambda}: C_{0}^{\lambda}=\operatorname{Hom}\left(M^{\lambda}, k\right) \rightarrow \operatorname{Hom}\left(S^{\lambda}, k\right)=: C_{-1}^{\lambda},\left.\epsilon \mapsto \epsilon\right|_{S^{\lambda}} .
$$

In Proposition 3.3, we will show that $d_{0} \circ d_{1}=0$. Therefore, we obtain a chain complex

$$
\tilde{C}_{*}^{\lambda}: \quad 0 \rightarrow C_{a}^{\lambda} \stackrel{d_{a}}{\longrightarrow} C_{a-1}^{\lambda} \stackrel{d_{a-1}}{\longrightarrow} \cdots \stackrel{d_{1}}{\longrightarrow} C_{0}^{\lambda} \stackrel{d_{0}}{\longrightarrow} C_{-1}^{\lambda} \longrightarrow 0,
$$


in the category of finitely generated left $k \mathfrak{S}_{r}$-modules.

Proposition 3.3 With the notation from Sect. 3.2 one has $d_{0} \circ d_{1}=0$.

Proof We may assume that $\lambda$ is a partition, since otherwise $S^{\lambda}=0$ and $d_{0}=0$. Let $\gamma=\left(\lambda^{(0)} \triangleleft \lambda^{(1)}\right) \in \Delta_{1}^{\lambda}$ (so $\left.\lambda^{(0)}=\lambda\right)$, let $T \in \mathcal{T}^{\wedge}\left(\lambda, \lambda^{(1)}\right)$ and let $\epsilon \in \operatorname{Hom}\left(S^{\lambda^{(1)}}, k\right)$. We need to show that $\left(\epsilon \circ \theta_{T}\right)\left(S^{\lambda}\right)=0$. So let $t \in \mathcal{T}^{\text {st }}(\lambda)$. It suffices to show that $\epsilon\left(\theta_{T}\left(e_{t}\right)\right)=0$. We can write $\theta_{T}\left(e_{t}\right)=\theta_{T}\left(\kappa_{t} t\right)=\kappa_{t} \theta_{T}(t)$ and $\theta_{T}(t)=s_{1}+\cdots+s_{a}$ with elements $s_{1}, \ldots, s_{a} \in \mathcal{T}^{\mathrm{rs}}\left(\lambda^{(1)}\right)$ according to the explicit rule in (2.12). Thus, it suffices to show that $\kappa_{t} \cdot s=0$ for all $s \in\left\{s_{1}, \ldots, s_{a}\right\}$.

Let $i$ be the smallest positive integer such that the $i$ th row of $T$ does not have all entries equal to $i$. Then, since $T \in \mathcal{T}^{\wedge}\left(\lambda, \lambda^{(1)}\right)$, the $i$ th row of $T$ contains an entry $j$ with $j>i$. By the explicit description of $\theta_{T}(t)$ in (2.12) we see that $s$ has the following property: The $i$ th row of $s$ consists of all the entries of the $i$ th row of $t$ and some additional entries, one of them being an entry $x$ from the $j$ th row of $t$. Since $\lambda$ is a partition, there exists an entry $y$ in the $i$ th row of $t$ which lies in the same column of $t$ as the entry $x$ in the $j$ th row of $t$. It follows that the transposition $\tau:=(x, y)$ is contained in $C_{t}$. Note that $\tau \cdot s=s$, since $x$ and $y$ are in the same row of $s$. If $\mathcal{R} \subset C_{t}$ denotes a set of representatives for $C_{t} /\{1, \tau\}$ then we obtain

$$
\kappa_{t} \cdot s=\sum_{\sigma \in \mathcal{R}} \operatorname{sgn}(\sigma) \sigma(1-\tau) \cdot s=0,
$$

since $(1-\tau) \cdot s=0$, and the proof is complete.

The chain complex $\tilde{C}_{*}^{\lambda}$ is not exact, in general. For example, if $\lambda=(1,3)$ its Euler characteristic is equal to 6 . But extensive computations in cases where $\lambda$ is a partition lead us to the following:

Conjecture 3.4 If $\lambda$ is a partition then $\tilde{C}_{*}^{\lambda}$ is exact.

Remark 3.5 (a) Using computer calculations, we verified that Conjecture 3.4 holds for all partitions $\lambda$ when $r \leq 5$.

(b) If we emphasize the base ring $k$ over which the chain complex $\tilde{C}_{*}^{\lambda}$ is constructed then we write ${ }_{k} \tilde{C}_{*}^{\lambda}$. If $\phi: k \rightarrow k^{\prime}$ is a homomorphism of commutative rings then it is easy to see from the construction of ${ }_{k} \tilde{C}_{*}^{\lambda}$ that

$$
k^{\prime} \otimes{ }_{k} \tilde{C}_{*}^{\lambda} \cong{ }_{k^{\prime}} \tilde{C}_{*}^{\lambda}
$$

as chain complexes of $k^{\prime} \mathfrak{S}_{r}$-modules. Therefore, in order to prove Conjecture 3.4 it suffices to show that ${ }_{\mathbb{Z}} \tilde{C}_{*}^{\lambda}$ is exact. In fact, if ${ }_{\mathbb{Z}} \tilde{C}_{*}^{\lambda}$ is exact then it splits as chain complex of $\mathbb{Z}$-modules, since all involved modules are free $\mathbb{Z}$-modules. Now, the isomorphism (3.3) applied to the canonical ring homomorphism $\phi: \mathbb{Z} \rightarrow k$ implies that ${ }_{k} \tilde{C}_{*}^{\lambda}$ is exact. 
3.6

For $n \geq 0$ we denote by $\mathcal{B}_{n}^{\lambda}$ the set of symbols

$$
\left(\lambda^{(0)} \underset{T_{1}}{\triangleleft} \lambda^{(1)} \underset{T_{2}}{\triangleleft} \cdots T_{T_{n}}^{\triangleleft} \lambda^{(n)}, t\right)
$$

with $\gamma=\left(\lambda=\lambda^{(0)} \triangleleft \cdots \triangleleft \lambda^{(n)}\right) \in \Delta_{n}^{\lambda}$ and $T_{i} \in \mathcal{T}^{\wedge}\left(\lambda^{(i)}, \lambda^{(i-1)}\right)$ for $i=1, \ldots, n$, and $t \in \mathcal{T}^{\mathrm{rs}}\left(\lambda^{(n)}\right)$. To each symbol as above we associate the element

$$
\theta_{T_{1}} \otimes \cdots \otimes \theta_{T_{n}} \otimes \epsilon_{t} \in M_{\gamma} \subseteq C_{n}^{\lambda}
$$

This way, the elements in $\mathcal{B}_{n}^{n}$ parametrize a $k$-basis of $C_{n}^{\lambda}$. If $n=0$ we have $\mathcal{B}_{0}^{\lambda}=$ $\mathcal{T}^{\mathrm{rs}}(\lambda)$.

By the definition of $d_{n}^{\lambda}$, we have for $n \geq 2$ and any symbol (3.4) in $\mathcal{B}_{n}^{\lambda^{(0)}}$ the recursion formula

$$
\begin{aligned}
& d_{n}^{\lambda^{(0)}}\left(\theta_{T_{1}} \otimes \cdots \otimes \theta_{T_{n}} \otimes \epsilon_{t}\right) \\
& \quad=\left(\theta_{T_{2}} \circ \theta_{T_{1}}\right) \otimes \cdots \otimes \theta_{T_{n}} \otimes \epsilon_{t}-\theta_{T_{1}} \otimes d_{n-1}^{\lambda^{(1)}}\left(\theta_{T_{2}} \otimes \cdots \otimes \theta_{T_{n}} \otimes \epsilon_{t}\right) .
\end{aligned}
$$

Finally, by $\phi_{t} \in \operatorname{Hom}\left(S^{\lambda}, k\right), t \in \mathcal{T}^{\mathrm{st}}(\lambda)$, we denote the dual $k$-basis of the basis $e_{t}$, $t \in \mathcal{T}^{\text {st }}(\lambda)$, of $S^{\lambda}$. For completeness, we set $\mathcal{B}_{-1}^{\lambda}:=\mathcal{T}^{\text {st }}(\lambda)$ and associate to $t \in \mathcal{B}_{-1}^{\lambda}$ the element $\phi_{t} \in \tilde{C}_{-1}^{\lambda}$.

\section{Exactness in degrees -1 and 0}

In this section, we prove that the chain complex $\tilde{C}_{*}^{\lambda}$ is exact in degrees -1 and 0 for all compositions $\lambda \in \Gamma_{r}$, cf. Theorem 4.2 and Theorem 4.3.

\section{1}

We will use the following strategy to prove exactness. Assume that for fixed $\lambda \in \Gamma_{r}$ and $n \geq-1$ we can find a subset $\mathcal{K}_{n}^{\lambda}$ of $\mathcal{B}_{n}^{\lambda}$ such that

$$
\operatorname{im}\left(d_{n+1}^{\lambda}\right)+K_{n}^{\lambda}=C_{n}^{\lambda}
$$

and

$$
\operatorname{ker}\left(d_{n}^{\lambda}\right) \cap K_{n}^{\lambda}=0
$$

hold, where $K_{n}^{\lambda}$ denotes the $k$-span of the basis elements of $C_{n}^{\lambda}$ parametrized by $\mathcal{K}_{n}^{\lambda}$. Then, we claim, the chain complex $\tilde{C}_{*}^{\lambda}$ is exact in degree $n$. In fact, let $c_{n} \in \operatorname{ker}\left(d_{n}^{\lambda}\right)$. Then, by $\left(A_{n}^{\lambda}\right)$, there exists $c_{n+1} \in C_{n+1}^{\lambda}$ and $x_{n} \in K_{n}^{\lambda}$ such that $c_{n}=d_{n+1}^{\lambda}\left(c_{n+1}\right)+x_{n}$. Applying $d_{n}^{\lambda}$ we obtain $d_{n}^{\lambda}\left(x_{n}\right)=d_{n}^{\lambda}\left(c_{n}\right)=0$, and, by $\left(B_{n}^{\lambda}\right)$, we have $x_{n}=0$. Thus, $c_{n}=d_{n+1}^{\lambda}\left(c_{n+1}\right) \in \operatorname{im}\left(d_{n+1}^{\lambda}\right)$, and we have exactness. Note that if $\left(A_{n}^{\lambda}\right)$ and $\left(B_{n}^{\lambda}\right)$ are satisfied then $K_{n}^{\lambda}$ is a complement of $\operatorname{ker}\left(d_{n}^{\lambda}\right)=\operatorname{im}\left(d_{n+1}^{\lambda}\right)$ in $C_{n}^{\lambda}$. 
Theorem 4.2 For every $\lambda \in \Gamma_{r}$, the empty subset $\mathcal{K}_{-1}^{\lambda}:=\emptyset$ of $\mathcal{B}_{-1}^{\lambda}$ satisfies $\left(A_{-1}^{\lambda}\right)$ and $\left(B_{-1}^{\lambda}\right)$. In particular, $\tilde{C}_{*}^{\lambda}$ is exact in degree -1 for every composition $\lambda$ of $r$.

Proof Since $K_{-1}^{\lambda}=0$, Condition $\left(B_{-1}^{\lambda}\right)$ is trivially satisfied, and Condition $\left(A_{-1}^{\lambda}\right)$ is equivalent to showing that the restriction map $d_{0}^{\lambda}: \operatorname{Hom}\left(M^{\lambda}, k\right) \rightarrow \operatorname{Hom}\left(S^{\lambda}, k\right)$ is surjective. If $\lambda$ is not a partition then $S^{\lambda}=0$ and this clearly holds. If $\lambda$ is a partition then $S^{\lambda}$ has a complement as $k$-submodule of $M^{\lambda}$. In fact, by (2.7), the element $e_{t}$ involves $t$ with coefficient 1 , and every other $t^{\prime} \in \mathcal{T}^{\text {st }}(\lambda)$ occurring with non-zero coefficient in $e_{t}$ is strictly larger than $t$ in the lexicographic order. This implies that the $k$-span of the elements $t \in \mathcal{T}^{\mathrm{rs}}(\lambda) \backslash \mathcal{T}^{\text {st }}(\lambda)$ forms a complement of $S^{\lambda}$ in $M^{\lambda}$. Therefore, every $k$-module homomorphism from $S^{\lambda}$ to $k$ can be extended to $M^{\lambda}$.

Theorem 4.3 Let $\lambda \in \Gamma_{r}$ and set

$$
\mathcal{K}_{0}^{\lambda}:=\left\{(\lambda, t) \in \mathcal{B}_{0}^{\lambda} \mid t \in \mathcal{T}^{\text {st }}(\lambda)\right\} .
$$

Then $\left(A_{0}^{\lambda}\right)$ and $\left(B_{0}^{\lambda}\right)$ are satisfied. In particular, $\tilde{C}_{*}^{\lambda}$ is exact in degree 0 for every composition $\lambda$ of $r$.

For the proof of property $\left(A_{0}^{\lambda}\right)$ in Theorem 4.3 we will need the following lemma. For $\lambda \in \Gamma_{r}$ and for any $t \in \mathcal{T}^{\mathrm{rs}}(\lambda)$ we denote by $C_{0,<t}^{\lambda}$ the $k$-span of the basis elements $\epsilon_{s} \in \operatorname{Hom}\left(M^{\lambda}, k\right)$ with $s \in \mathcal{T}^{\mathrm{rs}}(\lambda)$ satisfying $s<t$, cf. Sects. 3.2 and 2.2 for the definition of $\epsilon_{s}$ and for the lexicographic order $<$ on $\mathcal{T}(\lambda)$.

Lemma 4.4 Let $t \in \mathcal{T}^{\mathrm{rs}}(\lambda) \backslash \mathcal{T}^{\mathrm{st}}(\lambda)$. Then $\epsilon_{t} \in \operatorname{im}\left(d_{1}^{\lambda}\right)+C_{0,<t}^{\lambda}$.

Proof Since $t \notin \mathcal{T}^{\text {st }}(\lambda)$, there exist two consecutive rows in $t$, say row $q$ and row $q+1$, whose lengths $m:=\lambda_{q}$ and $n:=\lambda_{q+1}$ and entries

$$
\begin{array}{llll}
a_{1} & a_{2} & \cdots & a_{m} \\
b_{1} & b_{2} & \cdots & b_{n}
\end{array}
$$

satisfy

$$
\begin{array}{ll}
a_{1}<b_{1} & \text { and } \quad a_{2}<b_{2} \quad \text { and } \quad \cdots \quad \text { and } \\
a_{i}<b_{i} & \text { and } \quad\left(a_{i+1}>b_{i+1} \text { or } m=i\right)
\end{array}
$$

for some $i \in\{0,1, \ldots, \min \{m, n-1\}\}$. In other words, $i+1$ is the first position where either $a_{i+1}>b_{i+1}$ or the $(q+1)$ th row has an entry while the $q$ th row doesn't have an entry.

For any subset $X \subseteq\left\{a_{1}, \ldots, a_{m}, b_{1}, \ldots, b_{n}\right\}$ with at most $n$ elements, we denote by $t_{X}$ the tableau with the following property. The row lengths and entries of $t_{X}$ are the same as the row lengths and entries of $t$, except for rows $q$ and $q+1$. The $(q+1)$ th row of $t_{X}$ has the entries from $X$ in increasing order and the $q$ th row of $t_{X}$ has the 
entries from $\left\{a_{1}, \ldots, a_{m}, b_{1}, \ldots, b_{n}\right\} \backslash X$ in increasing order. Thus, $t_{X}$ has shape $\mu_{X}$ with $\left(\mu_{X}\right)_{j}=\lambda_{j}$ for all $j \notin\{q, q+1\},\left(\mu_{X}\right)_{q}=m+n-|X|$ and $\left(\mu_{X}\right)_{q+1}=|X|$. If $|X|<n$ then we have $\lambda \triangleleft \mu_{X}$ and $t_{X} \in \mathcal{T}^{\mathrm{rs}}\left(\mu_{X}\right)$. If $|X|=n$ then $\mu_{X}=\lambda$. Moreover, for $X$ as above, we denote by $T_{X} \in \mathcal{T}^{\wedge}\left(\mu_{X}, \lambda\right)$ the generalized tableau of shape $\mu_{X}$ and content $\lambda$ with all its $\lambda_{j}$ entries equal to $j$ in rows $j \notin\{q, q+1\}$, and with rows $q$ and $q+1$ of the form

$$
\begin{aligned}
& q \cdots q q+1 \cdots q+1 \\
& q+1 \cdots q+1
\end{aligned}
$$

with $\lambda_{q}$ entries equal to $q$. With this notation, we set

$$
v:=\sum_{Y \subseteq\left\{a_{1}, \ldots, a_{i}\right\}}(-1)^{|Y|} \theta_{T_{Y \cup\left\{b_{i+2}, \ldots, b_{n}\right\}}} \otimes \epsilon_{t_{Y \cup\left\{b_{i+2}, \ldots, b_{n}\right\}}} \in C_{1}^{\lambda} .
$$

Then we have

$$
\begin{aligned}
d_{1}^{\lambda}(v) & =\sum_{Y \subseteq\left\{a_{1}, \ldots, a_{i}\right\}}(-1)^{|Y|} \sum_{\substack{Y \cup\left\{b_{i+2}, \ldots, b_{n}\right\} \subseteq X \subseteq\left\{a_{1}, \ldots, a_{m}, b_{1}, \ldots, b_{n}\right\} \\
|X|=n}} \epsilon_{t_{X}} \sum_{\substack{\left\{b_{i+2}, \ldots, b_{n}\right\} \subseteq X \subseteq\left\{a_{1}, \ldots, a_{m}, b_{1}, \ldots, b_{n}\right\} \\
|X|=n}}\left(\sum_{Y \subseteq\left\{a_{1}, \ldots, a_{i}\right\} \cap X}(-1)^{|Y|}\right) \epsilon_{t_{X}} .
\end{aligned}
$$

The first of the above equations follows from (3.2). We analyze the inner sum and distinguish three cases for the set $X$.

If $X=\left\{b_{1}, \ldots, b_{n}\right\}$ then $t_{X}=t$, and the inner sum (over $Y$ ) is equal to 1 , since only $Y=\emptyset$ occurs. This case contributes the summand $\epsilon_{t}$ to the whole sum.

If $\left\{a_{1}, \ldots, a_{i}\right\} \cap X \neq \emptyset$ then the inner sum (over $Y$ ) vanishes, by an easy induction argument. This case contributes 0 to the whole sum.

If $\left\{a_{1}, \ldots, a_{i}\right\} \cap X=\emptyset$ but $X \neq\left\{b_{1}, \ldots, b_{n}\right\}$ then $t_{X}<t$. In fact, note that in this case $m \geq i+1$, and let $j \in\{1, \ldots, i+1\}$ be minimal with $b_{j} \notin X$. Then the $q$ th row of $t_{X}$ contains $a_{1}, \ldots, a_{i}$ and $b_{j}$, but not $b_{1}, \ldots, b_{j-1}$. Since

$$
a_{j-1}<b_{j-1}<b_{j} \leq b_{i+1}<a_{i+1},
$$

the $q$ th row of $t_{X}$ must start with

$$
a_{1} \ldots a_{p} b_{j} \ldots,
$$

where $p \in\{j-1, \ldots, i\}$ is maximal with $a_{p}<b_{j}$. Since $b_{j}<a_{p+1}$, we have $t_{X}<t$. Therefore, this case contributes an element of $C_{0,<t}^{\lambda}$ to the whole sum.

Altogether we obtain $\epsilon_{t} \in d_{1}^{\lambda}(v)+C_{0,<t}^{\lambda}$.

Proof of Theorem 4.3 We number the elements of $\mathcal{T}^{\mathrm{rs}}(\lambda)$ in increasing order: $t_{1}<$ $t_{2}<\cdots<t_{m}$. First, we show that $\left(A_{0}^{\lambda}\right)$ holds. For every $i \in\{1, \ldots, m\}$, we have either $\epsilon_{t_{i}} \in K_{0}^{\lambda}$ or $t_{i}$ is not standard. Using Lemma 4.4, it is straightforward to show that $\epsilon_{t_{i}} \in K_{0}^{\lambda}+\operatorname{im}\left(d_{1}^{\lambda}\right)$ by induction on $i$. 
Next we verify Condition $\left(B_{0}^{\lambda}\right)$. Assume that $\epsilon=\sum_{t \in \mathcal{T}^{\mathrm{st}}(\lambda)} \alpha_{t} \epsilon_{t} \in K_{0}^{\lambda} \subseteq$ $\operatorname{Hom}\left(M^{\lambda}, k\right)$ with $0=d_{0}^{\lambda}(\epsilon)=\left.\epsilon\right|_{S^{\lambda}}$ and assume that $\epsilon \neq 0$. Then $\lambda$ is a partition. Let $t_{*} \in \mathcal{T}^{\text {st }}(\lambda)$ be the largest tableau with $\alpha_{t_{*}} \neq 0$. Then, by (2.7), $\alpha_{t_{*}}=\epsilon\left(e_{t_{*}}\right)=0$, a contradiction.

Remark 4.5 (a) Calculations show that in general, for the set $\mathcal{K}_{n}^{\lambda}$ consisting of those symbols whose last entry $t$ is a standard tableau, the condition $\left(B_{n}^{\lambda}\right)$ does not hold. This already fails for $\lambda=(1,1,1,1)$. But one can show that $\left(A_{n}^{\lambda}\right)$ holds for this choice of $\mathcal{K}_{n}^{\lambda}$, for every composition $\lambda$. Thus, $K_{n}^{\lambda}$ covers $C_{n}^{\lambda} / \operatorname{im}\left(d_{n+1}^{\lambda}\right)$ for this choice, but it is too big to have trivial intersection with $\operatorname{ker}\left(d_{n}^{\lambda}\right)$. For $n=1$, our calculations of examples convince us that considering all symbols $\underset{T}{\triangleleft} \mu, t)$ with $\mu$ a partition, $t$ a standard tableau and $T$ a generalized standard tableau, conditions $\left(A_{1}^{\lambda}\right)$ and $\left(B_{1}^{\lambda}\right)$ will be satisfied for all partitions $\lambda$. However, we cannot prove this statement. Looking at larger values of $n$, computations show again that choosing $\mathcal{K}_{n}^{\lambda}$ by selecting those symbols with $t$ being a standard tableau and $T_{n}$ being a standard generalized tableau, conditions $\left(A_{n}^{\lambda}\right)$ and $\left(B_{n}^{\lambda}\right)$ will not be satisfied. It seems that one needs to require conditions on all generalized tableaux involved in the symbol. But we have no idea what these conditions should be.

(b) We have been considering the question if we can prove that the Lefschetz character of $\tilde{C}_{*}^{\lambda}$ over a field of characteristic 0 always vanishes. This is a necessary condition for the chain complex to be exact. Again, we are not able to prove this, but can show with the help of the computer algebra systems MAGMA and GAP that the Lefschetz character vanishes for all partitions $\lambda$ of $r$ with $r \leq 9$.

\section{Quasi-partitions and tame compositions}

\section{1}

To every composition $\lambda \in \Gamma_{r}$, one can associate two partitions in a natural way. The first arises by reordering the parts of $\lambda$ such that they are ordered by size. This partition will be denoted by $\lambda^{*}$. Obviously, one has $\lambda \unlhd \lambda^{*}$. Secondly, we can consider the set $\Lambda_{r}^{\unrhd \lambda}$ of all partitions $\mu$ of $r$ with $\mu \unrhd \lambda$. From Lemma 5.2, we can see that $\Lambda_{r}^{\triangleright \lambda}$ has a unique smallest element with respect to the dominance partial order. We will denote this element by $\bar{\lambda}$ and we will call it the closure of $\lambda$ in $\Lambda_{r}$. By definition, we have $\lambda \unlhd \bar{\lambda} \unlhd \lambda^{*}$, and we say that $\lambda$ is a quasi-partition if $\bar{\lambda}=\lambda^{*}$. Clearly, if $\lambda$ is a partition then $\lambda=\bar{\lambda}=\lambda^{*}$ and $\lambda$ is also a quasi-partition. We will denote the set of quasi-partitions of $r$ by $\widetilde{\Lambda}_{r}$. Thus, $\Lambda_{r} \subseteq \widetilde{\Lambda}_{r} \subseteq \Gamma_{r}$.

Lemma 5.2 (a) Let $\lambda \in \Gamma_{r}$ be a composition which is not a partition and assume that $\mu \in \Lambda_{r}$ is a partition with $\lambda \unlhd \mu$. Let $i$ be a positive integer with $\lambda_{i+1}>\lambda_{i}$ and define

$$
\tilde{\lambda}:=\left(\lambda_{1}, \ldots, \lambda_{i-1}, \lambda_{i}+1, \lambda_{i+1}-1, \lambda_{i+2}, \ldots\right) .
$$

Then $\lambda \triangleleft \tilde{\lambda} \unlhd \mu$.

(b) Let $\lambda \in \Gamma_{r}$ be a composition. The set of partitions of $r$ which dominate $\lambda$ has a unique minimal element with respect to the dominance partial order. 
Proof (a) Clearly, we have $\lambda \triangleleft \tilde{\lambda}$. Note that the $m$ th partial sum $\sum_{j=1}^{m} \tilde{\lambda}_{j}$ coincides with the $m$ th partial sum of $\lambda$ for all $m$ except for $m=i$. Thus, in order to show that $\tilde{\lambda} \unlhd \mu$ it suffices to show that the $i$ th partial sum of $\tilde{\lambda}$ is less or equal to the $i$ th partial sum of $\mu$. So assume that $\lambda_{1}+\lambda_{2}+\cdots+\lambda_{i}+1>\mu_{1}+\cdots+\mu_{i}$. Then $\lambda_{1}+\cdots+\lambda_{i} \geq \mu_{1}+\cdots+\mu_{i}$, which forces equality since $\mu$ dominates $\lambda$. Again, since $\mu$ dominates $\lambda$, this forces $\lambda_{m}=\mu_{m}$ for all $m=1, \ldots, i$. But $\lambda_{i+1}>\lambda_{i}=\mu_{i} \geq \mu_{i+1}$, since $\mu$ is a partition. This implies that the $(i+1)$ th partial sum of $\lambda$ is bigger than the $(i+1)$ th partial sum of $\mu$. This is a contradiction. And we have shown that $\tilde{\lambda} \unlhd \mu$.

(b) If $\lambda$ is a partition the statement obviously holds. So assume that $\lambda$ is not a partition. Note that the set of compositions of $r$ that dominate $\lambda$ is a finite set and that it contains a partition (namely $(r)$ ). Therefore, the process of replacing $\lambda$ by $\tilde{\lambda}$ as in Part (a) will produce a partition after finitely many steps. By Part (a), this partition is dominated by every partition that dominates $\lambda$.

Remark 5.3 The proof of Lemma 5.2 shows that the closure $\bar{\lambda}$ of a composition $\lambda \in$ $\Gamma_{r}$ can be constructed by repeating the process $\lambda \mapsto \tilde{\lambda}$ until one reaches a partition. Here, $\tilde{\lambda}$ is not determined by $\lambda$, since there might be several indices $i$ as in part (a) of the lemma. But all choices lead to the same partition $\bar{\lambda}$.

We will need the following well-known result about $\lambda^{*}$. A proof using the RSK algorithm can be found in [8, Sect. 4.3, Proposition 2], for instance. We include a short elementary proof.

Proposition 5.4 Let $\lambda \in \Gamma_{r}$ be a composition and let $\mu \in \Lambda_{r}$ be a partition. Then

$$
\left|\mathcal{T}^{\mathrm{st}}(\mu, \lambda)\right|=\left|\mathcal{T}^{\mathrm{st}}\left(\mu, \lambda^{*}\right)\right|
$$

Proof Let $\lambda=\left(\lambda_{1}, \ldots, \lambda_{n}\right)$ and $\sigma \cdot \lambda:=\left(\lambda_{\sigma^{-1}(1)}, \ldots, \lambda_{\sigma^{-1}(n)}\right)$ for some $\sigma \in \mathfrak{S}_{n}$. We will show that

$$
\left|\mathcal{T}^{\mathrm{st}}(\mu, \lambda)\right|=\left|\mathcal{T}^{\mathrm{st}}(\mu, \sigma \cdot \lambda)\right| \text {. }
$$

For that, we can assume that $\sigma=(i, i+1)$ for some $1 \leq i \leq n-1$. In this case, we will construct the following map from $\mathcal{T}^{\text {st }}(\mu, \lambda)$ to $\mathcal{T}^{\text {st }}(\mu, \sigma \cdot \lambda)$. In every row of a generalized tableau $T \in \mathcal{T}^{\text {st }}(\mu, \lambda)$, we leave all boxes unchanged that have entries different from $i$ and $i+1$. The entries that are equal to $i$ or $i+1$ occupy a connected string $S$ of boxes in this row. The boxes with entry $i$ which have boxes with entry $i+1$ below them we leave unchanged. These boxes form a connected string $S^{\prime}$ at the left end of $S$. Similarly, the boxes with entry $i+1$ which have boxes with entry $i$ above them we leave unchanged. These boxes form a connected string $S^{\prime \prime}$ at the right end of $S$. If the middle part $S \backslash\left(S^{\prime} \cup S^{\prime \prime}\right)$ of the string $S$ consists of $a$ boxes with entry $i$ and $b$ boxes with entry $i+1$, we replace these entries with $b$ entries equal to $i$ and $a$ entries equal to $i+1$ (in ascending order). It is clear that the result is a generalized standard tableau of the same shape and with content $\sigma \cdot \lambda$. Applying the same construction to an element of $\mathcal{T}^{\text {st }}(\mu, \sigma \cdot \lambda)$ results in an element of $\mathcal{T}^{\text {st }}(\mu, \lambda)$ and the two maps are inverse to each other. 
Lemma 5.5 Assume that $\lambda \in \widetilde{\Lambda}_{r}$ is a quasi-partition and set

$$
\left.\mathcal{K}_{1}^{\lambda}:=\{\underset{T}{\triangleleft} \mu, t) \in \mathcal{B}_{1}^{\lambda} \mid t \text { standard and } T \text { standard }\right\} .
$$

Then

$$
\left|\mathcal{K}_{1}^{\lambda}\right|=\left|\mathcal{T}^{\mathrm{rs}}(\lambda)\right|-\left|\mathcal{T}^{\mathrm{st}}(\lambda)\right|
$$

Proof We have

$$
\begin{aligned}
\left|\mathcal{K}_{1}^{\lambda}\right| & =\sum_{\lambda \triangleleft \mu \in \Gamma_{r}}\left|\mathcal{T}^{\mathrm{st}}(\mu, \lambda)\right| \cdot\left|\mathcal{T}^{\mathrm{st}}(\mu)\right| \\
& =\sum_{\lambda \triangleleft \mu \in \Lambda_{r}}\left|\mathcal{T}^{\mathrm{st}}(\mu, \lambda)\right| \cdot\left|\mathcal{T}^{\mathrm{st}}(\mu)\right| .
\end{aligned}
$$

Here, the second equation holds since $\mathcal{T}^{\text {st }}(\mu)=\emptyset$ if $\mu \in \Gamma_{r} \backslash \Lambda_{r}$. If $\lambda$ is a partition then the last expression in (5.1) equals $\mathrm{rk} M^{\lambda}-\mathrm{rk} S^{\lambda}$, by Young's rule (2.8). If $\lambda$ is not a partition then $\mathrm{rk} S^{\lambda}=0$ and $\left|\mathcal{T}^{\mathrm{st}}(\mu, \lambda)\right|=\left|\mathcal{T}^{\mathrm{st}}\left(\mu, \lambda^{*}\right)\right|$, by Proposition 5.4. Since $\lambda$ is a quasi-partition, this implies that the last expression in (5.1) equals

$$
\sum_{\lambda^{*} \unlhd \mu \in \Lambda_{r}} \kappa_{\lambda^{*}, \mu} \cdot \operatorname{rk} S^{\mu}=\operatorname{rk} M^{\lambda^{*}}=\operatorname{rk} M^{\lambda}=\operatorname{rk} M^{\lambda}-\operatorname{rk} S^{\lambda} .
$$

Thus, altogether we obtain in both cases

$$
\left|\mathcal{K}_{1}^{\lambda}\right|=\operatorname{rk} M^{\lambda}-\operatorname{rk} S^{\lambda}=\left|\mathcal{T}^{\mathrm{rs}}(\lambda)\right|-\left|\mathcal{T}^{\mathrm{st}}(\lambda)\right|,
$$

and the proof is complete.

We call a composition $\lambda \in \Gamma_{r}$ tame if every composition $\mu \in \Gamma_{r}$ with $\lambda \unlhd \mu$ is a quasi-partition. This is the technical condition that will allow us to prove Theorem 6.1 by induction. In order to know more precisely to which compositions this theorem applies, we want to determine tame composition explicitly in part (a) of the next proposition. Part (b) gives a crucial property of tame compositions that is also used in the proof of Theorem 6.1. Note that if $\lambda$ is tame then also every composition dominating $\lambda$ is tame.

Proposition 5.6 Let $\lambda=\left(\lambda_{1}, \lambda_{2}, \ldots\right) \in \Gamma_{r}$ be a composition.

(a) The composition $\lambda$ is tame if and only if one of the following holds:

(i) $\lambda_{3}+\lambda_{4}+\cdots=0$ and $\lambda_{1}+1 \geq \lambda_{2}$.

(ii) $\lambda_{3}+\lambda_{4}+\cdots=1$ and $\lambda_{1} \geq \lambda_{2}$.

(b) If $\lambda$ is tame then $\mathcal{T}^{\wedge}(\mu, \lambda)=\mathcal{T}^{\text {st }}(\mu, \lambda)$ for every partition $\mu \in \Lambda_{r}$ with $\lambda \triangleleft \mu$.

Proof Assume that $\lambda$ is tame. We first show that $\lambda_{3}+\lambda_{4}+\cdots \leq 1$. Assume that $\lambda_{3}+\lambda_{4}+\cdots \geq 2$. Then $\lambda \unlhd(r-2,0,2)=: \mu$. But $\mu$ is not a quasi-partition. In fact, if $r-2=0$ then $\mu^{*}=(2)$ and $\bar{\mu}=(1,1)$, if $r-2=1$ then $\mu^{*}=(2,1)$ and $\bar{\mu}=$ $(1,1,1)$, and if $r-2 \geq 2$ then $\mu^{*}=(r-2,2)$ and $\bar{\mu}=(r-2,1,1)$. This contradicts 
the tameness of $\lambda$, and therefore $\lambda_{3}+\lambda_{4}+\cdots \leq 1$. Now we consider the case that $\lambda_{3}+\lambda_{4}+\cdots=1$. We will show that $\lambda_{1} \geq \lambda_{2}$. Assume that $\lambda_{1}<\lambda_{2}$. Then $\mu:=$ $\left(\lambda_{1}, \lambda_{2}+1\right) \triangleright \lambda$ and $\mu$ satisfies $\mu_{2}-\mu_{1} \geq 2$. Therefore, $\mu^{*}=\left(\mu_{2}, \mu_{1}\right)$ and $\tilde{\mu}:=$ $\left(\mu_{2}-1, \mu_{1}+1\right)$ is a partition satisfying $\mu \unlhd \tilde{\mu} \triangleleft \mu^{*}$. This implies $\bar{\mu} \unlhd \tilde{\mu} \triangleleft \mu^{*}$, contradicting the tameness of $\lambda$. Finally, we consider the case that $\lambda_{3}+\lambda_{4}+\cdots=0$. Then $\lambda=\left(\lambda_{1}, \lambda_{2}\right)$. Assume that $\lambda_{1}+1<\lambda_{2}$. Then $\lambda_{2}-\lambda_{1} \geq 2$ and $\lambda$ is not a quasipartition (as we have seen for $\mu$ in the previous case). Therefore, $\lambda_{1}+1 \geq \lambda_{2}$ in this case. This finishes the proof of the forward implication in part (a).

Next assume that $\lambda$ satisfies (i). Then the compositions that dominate $\lambda$ are of the form $\lambda^{(i)}=\left(\lambda_{1}+i, \lambda_{2}-i\right)$ for $i=0, \ldots, \lambda_{2}$, and this set is totally ordered. Clearly, $\lambda^{(i)}$ is a partition for $i=1, \ldots, \lambda_{2}$. Also $\lambda=\lambda^{(0)}$ is a partition unless $\lambda_{2}=\lambda_{1}+1$. In this case, $\lambda^{*}=\left(\lambda_{2}, \lambda_{1}\right)=\bar{\lambda}$. Therefore, $\lambda$ is tame. Moreover, for $i \in\left\{1, \ldots, \lambda_{2}\right\}$, we see immediately that there exists only one element $T$ in $\mathcal{T}^{\wedge}\left(\lambda^{(i)}, \lambda\right)$, namely the generalized tableaux with $\lambda_{1}$ entries equal to 1 in the first row followed by $i$ entries equal to 2 in the first row and $\lambda_{2}-i$ entries equal to 2 in the second row. Since $\lambda_{1} \geq \lambda_{2}-i$, this generalized tableau is also standard. This shows that the reverse implication of part (a) and also that part (b) holds if $\lambda$ is of the form (i).

Next we assume that $\lambda$ satisfies (ii). The compositions that dominate $\lambda$ are of the form $\mu=\left(\mu_{1}, \mu_{2}, \ldots\right)$ with either $\mu$ being of type (i) with $\lambda_{1} \leq \mu_{1}$, or $\mu$ being of type (ii) with $\mu_{1} \geq \lambda_{1}$ and $\mu_{1}+\mu_{2}=r-1=\lambda_{1}+\lambda_{2}$.

First, we assume that $\mu$ is of type (i). Then $\mu$ is tame (and in particular a quasipartition) by the previous paragraph. Moreover, if $\mu$ is a partition and if $T \in \mathcal{T}^{\wedge}(\mu, \lambda)$ then all $\lambda_{1}$ entries of $T$ that are equal to 1 have to be in the first row of $T$. Therefore, $T$ is determined by the position of the single entry which is larger than 2 . This entry can be at the end of the first row or at the end of the second row. The only obstruction to $T$ being standard can occur if an entry equal to 2 in the second row is positioned below some entry $\geq 2$ in the first row. There are only $\lambda_{2}$ entries equal to 2 and $\lambda_{2} \leq \lambda_{1}$, with $\lambda_{1}$ the number of entries equal to 1 in the first row. This shows that $T$ is standard.

Finally, we assume that $\mu$ is of type (ii). Recall that $\mu_{1} \geq \lambda_{1} \geq \lambda_{2} \geq \mu_{2}$. We first show that $\mu$ is a quasi-partition. In fact, if $\mu_{1}=0$ then $\mu_{2}=0$ and $\mu^{*}=\bar{\mu}=(1)$; if $\mu_{1} \geq 1$ and $\mu_{2}=0$ then $\mu^{*}=\left(\mu_{1}, 1\right)=\bar{\mu}$; and if $\mu_{2} \geq 1$ then $\mu^{*}=\left(\mu_{1}, \mu_{2}, 1\right)=\bar{\mu}$. Now let $T \in \mathcal{T}^{\wedge}(\mu, \lambda)$ and assume that $\mu$ is a partition. This implies $\mu=\left(\mu_{1}, \mu_{2}, 1\right)$ with $\lambda_{1}+\lambda_{2}=\mu_{1}+\mu_{2}=r-1$. Since the $\lambda_{1}$ entries in $T$ that are equal to 1 are in the first row, the $\lambda_{2}$ entries equal to 2 fill the remaining positions in the first and second row, and the only entry larger than 2 must be in the third row. Since there are $\mu_{2}$ entries equal to 2 in the second row, and $\lambda_{1}$ entries equal to 1 in the first row and since $\mu_{2} \leq \lambda_{1}$, we see that $T$ is standard. This completes the proof of (a) and (b) in the case that $\lambda$ satisfies (ii).

\section{Exactness for tame compositions}

In this section, we prove the main theorem stating that $\tilde{C}_{*}^{\lambda}$ is exact for every tame composition. See Proposition 5.6 for a classification of tame compositions. 
Theorem 6.1 Let $\lambda \in \Gamma_{r}$ be a tame composition. For $n=-1$ set $\mathcal{K}_{-1}^{\lambda}=\emptyset$, for $n=0$ set $\mathcal{K}_{0}^{\lambda}=\mathcal{T}^{\mathrm{st}}(\lambda)$, and for $n \geq 1$ set

$$
\mathcal{K}_{n}^{\lambda}:=\left\{\left(\lambda=\lambda^{(0)} \underset{T_{1}}{\triangleleft}{ }_{T_{n}}^{\triangleleft} \lambda^{(n)}, t\right) \in \mathcal{B}_{n}^{\lambda} \mid t \in \mathcal{T}^{\mathrm{st}}\left(\lambda^{(n)}\right)\right\}
$$

Then $\left(A_{n}^{\lambda}\right)$ and $\left(B_{n}^{\lambda}\right)$ hold for all $n \geq-1$. In particular, the chain complex $\tilde{C}_{*}^{\lambda}$ is exact (cf. 4.1).

Proof We first show by induction on $n \in\{-1,0,1, \ldots\}$ that $\left(A_{n}^{\lambda}\right)$ holds. By Theorem 4.2 and Theorem 4.3, this is already shown for $n=-1$ and $n=0$. So let $n \geq 1$ and assume that $\left(A_{n-1}^{\mu}\right)$ holds for all tame $\mu \in \Gamma_{r}$. We will show that $\left(A_{n}^{\lambda}\right)$ holds for all tame $\lambda \in \Gamma_{r}$ by induction on the depth $d(\lambda)$ (cf. 3.2). If $d(\lambda)<n$ then $C_{n}^{\lambda}=0$ and $\left(A_{n}^{\lambda}\right)$ is satisfied. This anchors the induction proof. So assume that $\lambda \in \Gamma_{r}$ is tame and that $d(\lambda) \geq n$. We may assume that $\left(A_{n}^{\mu}\right)$ holds for all $\mu \in \Gamma_{r}$ with $\lambda \triangleleft \mu$, since then $\mu$ is also tame and $d(\mu)<d(\lambda)$. Let $\left(\lambda=\lambda^{(0)} \underset{T_{1}}{\triangleleft} \lambda_{T_{2}}^{(1)}{ }_{T_{T_{n}}}{ }^{\triangleleft} \lambda^{(n)}, t\right) \in \mathcal{B}_{n}^{\lambda}$. By induction on $n$ there exist elements $c_{n} \in C_{n}^{\lambda^{(1)}}$ and $x_{n-1} \in K_{n-1}^{\lambda^{(1)}}$ such that

$$
\theta_{T_{2}} \otimes \cdots \otimes \theta_{T_{n}} \otimes \epsilon_{t}=d_{n}^{\lambda^{(1)}}\left(c_{n}\right)+x_{n-1}
$$

Since $d\left(\lambda^{(1)}\right)<d(\lambda)$ we have $c_{n} \in K_{n}^{\lambda^{(1)}}+\operatorname{im}\left(d_{n+1}^{\lambda^{(1)}}\right)$ and we can write $c_{n}=x_{n}+$ $d_{n+1}^{\lambda^{(1)}}\left(c_{n+1}\right)$ with $x_{n} \in K_{n}^{\lambda^{(1)}}$ and $c_{n+1} \in C_{n+1}^{\lambda^{(1)}}$. Substituting $c_{n}$ in (6.1) yields

$$
\theta_{T_{2}} \otimes \cdots \otimes \theta_{T_{n}} \otimes \epsilon_{t}=d_{n}^{\lambda^{(1)}}\left(x_{n}\right)+x_{n-1}
$$

Applying (3.6) to the element $\theta_{T_{1}} \otimes x_{n} \in C_{n+1}^{\lambda}$ yields

$$
\begin{aligned}
d_{n+1}^{\lambda}\left(\theta_{T_{1}} \otimes x_{n}\right) & =y_{n}-\theta_{T_{1}} \otimes d_{n}^{\lambda^{(1)}}\left(x_{n}\right) \\
& =y_{n}-\theta_{T_{1}} \otimes \theta_{T_{2}} \otimes \cdots \otimes \theta_{T_{n}} \otimes \epsilon_{t}+\theta_{T_{1}} \otimes x_{n-1}
\end{aligned}
$$

with $y_{n} \in K_{n}^{\lambda}$, since $x_{n} \in K_{n}^{\lambda^{(1)}}$ and $\theta_{T_{1}} \otimes x_{n-1} \in K_{n}^{\lambda}$, since $x_{n-1} \in K_{n-1}^{\lambda^{(1)}}$. Therefore,

$$
\theta_{T_{1}} \otimes \theta_{T_{2}} \otimes \cdots \otimes \theta_{T_{n}} \otimes \epsilon_{t} \in K_{n}^{\lambda}+\mathrm{im}\left(d_{n+1}^{\lambda}\right)
$$

and $\left(A_{n}^{\lambda}\right)$ holds.

Next we show that $\left(B_{n}^{\lambda}\right)$ holds for all $n \geq-1$. We do this by showing the following stronger condition by induction on $n$ :

$$
\text { If } x \in K_{n}^{\lambda} \text { and } d_{n}^{\lambda}(x) \in K_{n-1}^{\lambda} \text { then } x=0 .
$$

If $n=-1$ there is nothing to show, since $K_{-1}^{\lambda}=0$. For $n=0$, condition $\left(\widetilde{B}_{0}^{\lambda}\right)$ is equivalent to condition $\left(B_{0}^{\lambda}\right)$, since $K_{-1}^{\lambda}=0$. Moreover, $\left(B_{0}^{\lambda}\right)$ is satisfied by Theorem 4.3. 
Next we show that $\left(\widetilde{B}_{1}^{\lambda}\right)$ holds. We denote by $N_{0}^{\lambda}$ the $k$-span of the basis elements of $C_{0}^{\lambda}$ which are parametrized by $\mathcal{B}_{0}^{\lambda} \backslash \mathcal{K}_{0}^{\lambda}$. Thus, $C_{0}^{\lambda}=K_{0}^{\lambda} \oplus N_{0}^{\lambda}$. We consider the $k$-linear map

$$
f: K_{1}^{\lambda} \stackrel{i}{\longrightarrow} C_{1}^{\lambda} \stackrel{d_{1}^{\lambda}}{\longrightarrow} C_{0}^{\lambda} \stackrel{p}{\longrightarrow} N_{0}^{\lambda}
$$

which is the composition of $d_{1}^{\lambda}$ with the inclusion $i: K_{1}^{\lambda} \rightarrow C_{1}^{\lambda}$ and the projection $p: C_{0}^{\lambda} \rightarrow N_{0}^{\lambda}$ with respect to the decomposition $C_{0}^{\lambda}=K_{0}^{\lambda} \oplus N_{0}^{\lambda}$. We will show that $f$ is an isomorphism. In order to see that $f$ is surjective, let $v_{0} \in N_{0}^{\lambda}$. By $\left(A_{0}^{\lambda}\right)$ we can write $v_{0}=d_{1}^{\lambda}\left(c_{1}\right)+x_{0}$ with $c_{1} \in C_{1}^{\lambda}$ and $x_{0} \in K_{0}^{\lambda}$, and by $\left(A_{1}^{\lambda}\right)$ we can write $c_{1}=d_{2}^{\lambda}\left(c_{2}\right)+x_{1}$ with $c_{2} \in C_{2}^{\lambda}$ and $x_{1} \in K_{1}^{\lambda}$. Substituting $c_{1}$ in the first equation yields $v_{0}=d_{1}^{\lambda}\left(x_{1}\right)+x_{0}$ and $d_{1}^{\lambda}\left(x_{1}\right)=v_{0}-x_{0}$. This implies that $f\left(x_{1}\right)=v_{0}$. Thus, $f$ is surjective. By Lemma 5.5, we have

$$
\operatorname{rk} K_{1}^{\lambda}=\left|\mathcal{T}^{\mathrm{rs}}(\lambda)\right|-\left|\mathcal{T}^{\mathrm{st}}(\lambda)\right|=\left|\mathcal{B}_{0}^{\lambda} \backslash \mathcal{K}_{0}^{\lambda}\right|=\operatorname{rk} N_{0}^{\lambda}
$$

Together with the surjectivity of $f$ this implies that $f$ is an isomorphism. In order to see that $\left(\widetilde{B}_{1}^{\lambda}\right)$ holds, let $x_{1} \in K_{1}^{\lambda}$ and assume that $d_{1}^{\lambda}\left(x_{1}\right) \in K_{0}^{\lambda}$. Then $f\left(x_{1}\right)=0$ and the injectivity of $f$ implies $x_{1}=0$. This completes the proof that $\left(\widetilde{B}_{1}^{\lambda}\right)$ holds.

Now let $n \geq 2$ and assume that $\left(\widetilde{B}_{n-1}^{\mu}\right)$ holds for every tame composition $\mu \in \Gamma_{r}$. Note that this applies in particular to every $\mu$ which dominates $\lambda$. Let $x_{n} \in K_{n}^{\lambda}$ and assume that $d_{n}^{\lambda}\left(x_{n}\right) \in K_{n-1}^{\lambda}$. We will show that $x_{n}=0$. We can write $x_{n}$ as a $k$-linear combination of the basis elements $\theta_{T_{1}} \otimes \cdots \otimes \theta_{T_{n}} \otimes \epsilon_{t}$ parametrized by the symbols $\left(\lambda^{(0)}{ }_{T_{1}} \lambda^{(1)}{ }_{T_{2}}^{\triangleleft} \cdots \triangleleft{ }_{T_{n}} \lambda^{(n)}, t\right) \in \mathcal{B}_{n}^{\lambda}$. Since a generalized tableau $T \in \mathcal{T}(\mu, \nu)$ determines $\mu$ and $\nu$, we will abbreviate the above symbol by $\left(T_{1}, T_{2}, \ldots, T_{n}, t\right)$ and we can write

$$
x_{n}=\sum_{\left(T_{1}, \ldots, T_{n}, t\right) \in \mathcal{K}_{n}^{\lambda}} a\left(T_{1}, \ldots, T_{n}, t\right) \cdot \theta_{T_{1}} \otimes \cdots \otimes \theta_{T_{n}} \otimes \epsilon_{t}
$$

with uniquely determined elements $a\left(T_{1}, \ldots, T_{n}, t\right) \in k$. We apply the formula from (3.6) to $x_{n}$ and obtain

$$
\begin{aligned}
d_{n}^{\lambda}\left(x_{n}\right)= & \sum_{\left(T_{1}, \ldots, T_{n}, t\right) \in \mathcal{K}_{n}^{\lambda}} a\left(T_{1}, \ldots, T_{n}, t\right) \cdot\left(\theta_{T_{2}} \circ \theta_{T_{1}}\right) \otimes \theta_{T_{3}} \otimes \cdots \otimes \theta_{T_{n}} \circ \epsilon_{t} \\
& -\sum_{\left(T_{1}, \ldots, T_{n}, t\right) \in \mathcal{K}_{n}^{\lambda}} a\left(T_{1}, \ldots, T_{n}, t\right) \cdot \theta_{T_{1}} \otimes d_{n-1}^{\lambda^{(1)}}\left(\theta_{T_{2}} \otimes \cdots \otimes \theta_{T_{n}} \otimes \epsilon_{t}\right),
\end{aligned}
$$

where $\lambda^{(1)}$ denotes the shape of $T_{1}$. Next we fix an element $\mu \in \Gamma_{r}$ with $\lambda \triangleleft \mu$ and an element $S \in \mathcal{T}^{\wedge}(\mu, \lambda)$. We define $\pi_{S}: K_{n-1}^{\lambda} \rightarrow K_{n-2}^{\lambda}$ as the $k$-linear map which sends a basis element parametrized by a symbol $\left(T_{1}, \ldots, T_{n-1}, t\right) \in \mathcal{K}_{n-1}^{\lambda}$ to the basis element parametrized by the symbol $\left(T_{2}, \ldots, T_{n-1}, t\right) \in \mathcal{K}_{n-2}^{\mu}$ if $T_{1}=S$ and to 0 otherwise. Note that both sides of (6.2) lie in $K_{n-1}^{\lambda}$ so that we can apply $\pi_{S}$. The 
application of $\pi_{S}$ to the second sum in (6.2) yields

$$
\sum_{\substack{\left(T_{1}, T_{2}, \ldots, T_{n}, t\right) \in \mathcal{K}_{n}^{\lambda} \\ T_{1}=S}} a\left(S, T_{2}, \ldots, T_{n}, t\right) \cdot d_{n-1}^{\mu}\left(\theta_{T_{2}} \otimes \cdots \otimes \theta_{T_{n}} \otimes \epsilon_{t}\right)=d_{n-1}^{\mu}\left(x_{n-1}^{S}\right),
$$

with

$$
x_{n-1}^{S}:=\sum_{\substack{\left(T_{1}, T_{2}, \ldots, T_{n}, t\right) \in \mathcal{K}_{n}^{\lambda} \\ T_{1}=S}} a\left(S, T_{2}, \ldots, T_{n}, t\right) \cdot \theta_{T_{2}} \otimes \cdots \otimes \theta_{T_{n}} \otimes \epsilon_{t} \in K_{n-1}^{\mu} .
$$

This implies that $d_{n-1}^{\mu}\left(x_{n-1}^{S}\right) \in K_{n-2}^{\mu}$. The induction hypothesis now implies $x_{n-1}^{S}=0$. But

$$
x_{n}=\sum_{\lambda \triangleleft \mu \in \Gamma_{r}} \sum_{S \in \mathcal{T}^{\wedge}(\mu, \lambda)} \theta_{S} \otimes x_{n-1}^{S},
$$

and we obtain $x_{n}=0$. This shows that $\left(\widetilde{B}_{n}^{\lambda}\right)$ and $\left(B_{n}^{\lambda}\right)$ hold and the proof is complete.

\section{Comparison with similar complexes}

We end our article with a short discussion of related results. All of these were obtained using quite different methods, working with representations of the general linear group instead. More precisely, one constructs a resolution of the Weyl module by symmetric powers of the standard representation. Then applying the Schur functor to this complex gives an exact complex of modules for the symmetric group, where the Weyl module is sent to the corresponding Specht module, and the symmetric powers are sent to permutation modules. Though our construction is different in nature, it is interesting to compare the results. To keep this section short, we just present results without any details on the construction. We refer the reader to the respective articles, and to [9] for details on the intimate connection between representations of the symmetric group and polynomial representations of the general linear group.

The first constructions of this kind were given independently by Akin [1] and Zelevinskii [14]. They both used the Bernstein-Gelfand-Gelfand resolution ([4]) to give a resolution of Weyl modules over the complex numbers. Zelevinskii also notes that one obtains a corresponding resolution for Specht modules in Example 3 at the end of [14]. There was no hope that this complex would be exact in positive characteristic, or even over any commutative ring. In fact, one easily checks that even for small partitions our resolution will involve many more terms than the Akin-Zelevinskii complex. For example, consider the partition $\lambda=(1,1,1)$. The Akin-Zelevinskii resolution

$$
0 \rightarrow M^{(3)} \rightarrow M^{(2,1)} \oplus M^{(2,1)} \rightarrow M^{(1,1,1)} \rightarrow S^{(1,1,1)} \rightarrow 0
$$

has length 2, while our resolution has length 3 and involves several more summands. 
Akin and Buchsbaum also realized that the complex must be extended to work in a characteristic-free setting. In [2] and [3], they work out such an extension over an arbitrary infinite field. However, an explicit form of the complex could only be obtained for two-part partitions (in fact, they allow not just partitions, but more general skew-shapes). It is easily seen that for two-part partitions our resolution has the same terms as the one given by Akin and Buchsbaum, namely

$$
C_{i}^{\left(\lambda_{1}, \lambda_{2}\right)}=\sum_{t=1}^{\lambda_{2}}\left(\begin{array}{l}
i-1 \\
t-1
\end{array}\right) \times M^{\left(\lambda_{1}+t, \lambda_{2}-t\right)},
$$

cf. Sect. 4 in [2].

There are other generalizations of the Akin-Zelevinskii resolution. In [6], Donkin works in the more general context of reductive algebraic groups and gives a new proof of existence of resolutions, but no explicit description. In a similar spirit, Doty [7] constructs resolutions for one-dimensional representations of the Borel subgroup of an algebraic group in characteristic zero. Also in characteristic zero, Woodcock [12] constructs resolutions for simple $S(B)$-modules, where $S(B)$ is the Schur algebra for the subgroup $B$ of lower triangular matrices in $G L_{n}(k)$. He also shows in [13] that such resolutions induce projective resolutions of Weyl modules. A similar approach is taken by Santana in [11]. She constructs minimal projective resolutions of simple $S(B)$-modules in characteristic zero. Over a field of positive characteristic she describes the first three terms of a minimal projective resolution when $n=2$.

While we are working in a more special situation, considering only the case of the group algebra of the symmetric group, our construction has other advantages. One is what we call integrability, i.e., the construction works over any commutative ring. Another one is functoriality, cf. Remark 3.5(b). Finally, the construction of the chain complex $\tilde{C}_{*}^{\lambda}$ is completely explicit and combinatorial. This allows us to actually compute resolutions using computer calculations.

Open Access This article is distributed under the terms of the Creative Commons Attribution Noncommercial License which permits any noncommercial use, distribution, and reproduction in any medium, provided the original author(s) and source are credited.

\section{References}

1. Akin, K.: On complexes relating the Jacobi-Trudi identity with the Bernstein-Gelfand-Gelfand resolution. J. Algebra 117, 494-503 (1988)

2. Akin, K., Buchsbaum, D.A.: Characteristic-free representation theory of the general linear group. Adv. Math. 58, 149-200 (1985)

3. Akin, K., Buchsbaum, D.A.: Characteristic-free representation theory of the general linear group, II: homological considerations. Adv. Math. 72, 171-210 (1988)

4. Bernstein, I.N., Gelfand, I.M., Gelfand, S.I.: Differential operators on the base affine space and a study of $\mathfrak{g}$-modules. In: Gelfand, I.M. (ed.) Lie Groups and Their Representations, pp. 21-64. Wiley, New York (1975)

5. Curtis, C.W., Reiner, I.: Methods of Representation Theory, vol. 1. Wiley, New York (1981)

6. Donkin, S.: Finite resolutions of modules for reductive algebraic groups. J. Algebra 101, 473-488 (1986)

7. Doty, S.R.: Resolutions of $B$ modules. Indag. Math. 5(3), 267-283 (1994) 
8. Fulton, W.: Young Tableaux. Cambridge University Press, Cambridge (1999)

9. Green, J.A.: Polynomial Representations of $G L_{n}$. Springer Lecture Notes, vol. 830. Springer, Berlin (1980)

10. James, G.D.: The Representation Theory of the Symmetric Groups. Lecture Notes in Mathematics, vol. 682. Springer, Berlin (1978)

11. Santana, A.P.: The Schur algebra $S\left(B^{+}\right)$and projective resolutions of Weyl modules. J. Algebra 161, 480-504 (1993)

12. Woodcock, D.J.: Borel Schur algebras. Commun. Algebra 22(5), 1703-1721 (1994)

13. Woodcock, D.J.: A vanishing theorem for Schur modules. J. Algebra 165, 483-506 (1994)

14. Zelevinskii, A.V.: Resolvents, dual pairs, and character formulas. Funct. Anal. Appl. 21, 152-154 (1987) 